\title{
Las traducciones al inglés de Le Deuxième Sexe de Simone de Beauvoir: Prostitutas y hetairas
}

\section{(The English Translations of Le Deuxième Sexe by Simone de Beauvoir: Prostitutes and Hetaeras)}

\author{
MARÍA LUISA RODRÍGUEZ MUÑOZ \\ Universidad de Córdoba \\ Ir1romum@uco.es
}

Fecha de recepción: 15 de junio de 2016

Fecha de aceptación: 28 de junio de 2016

Resumen: En el presente estudio cotejamos las dos traducciones al inglés existentes del capítulo sobre la prostitución del Segundo Sexo de Simone de Beauvoir con el original a fin de constatar las polémicas alteraciones de la primera versión anglosajona al completo y sus efectos. De esta forma, abordamos un análisis traductológico que, si bien parte de la descripción de todos los agentes del encargo y de las afirmaciones realizadas por filósofas como Moi (2002), se detiene en el aspecto microtextual de un corpus mucho más breve no estudiado en profundidad desde esta perspectiva bidimensional (proceso/producto).

Palabras clave: Ciencias sociales, Feminismo, Manipulación, Proceso y producto de traducción.

Abstract: The present study compares the two translations of the chapter on prostitution from The Second Sex by Simone de Beauvoir and its original French text to attest the controversial changes in its complete first English version and their effects. Thus we conduct a translation analysis based on the description of the agents involved in the translation assigment as well as on statements by philosohers such as Moi (2002). We also focus on the microtextual aspect of a brief corpus which has not been studied in depth from this bidimensional perspective (process/product).

Key words: Social sciences, Feminism, Manipulation, Translation process and product. 


\section{INTRODUCCIÓN}

En 1949 ve la luz una de los ensayos feministas de la historia de la humanidad más influyentes, El segundo sexo de Simone de Beauvoir. En 1953, se publica su traducción al inglés realizada por Howard M. Parshley, un zoólogo estadounidense experto en reproducción. La primera versión se comercializó hasta el 2009, momento en el que, tras el aluvión de críticas que desde los 80 se vertieron sobre su baja calidad, se reescribe la versión anglosajona. En el mismo año, Bogic defiende una tesis doctoral en la Universidad de Ottawa en la que desvela la complicada interacción entre el traductor inicial y los editores y la enorme presión a la que estuvo sometido por las cortapisas de estos últimos. La autora completó la visión de la traducción entendida como proceso además de como producto y superó la crítica despiadada a la que fue sometido el traductor obviando o menospreciando sus condiciones laborales.

Con la amplitud de miras que nos ofrece la nutrida bibliografía sobre el tema, nos proponemos señalar los puntos débiles de la traducción más longeva comparándola no solo con el original sino, por vez primera, con la nueva reescritura, mucho más fiel al estilo del texto de partida y que, asimismo, no escapa a las críticas por este rasgo literalista. Para ello, elegimos uno de los fragmentos no estudiados de forma aislada y pormenorizada hasta el momento y empleamos las categorías de errores y técnicas de traducción desde el punto de vista sintáctico, léxico y discursivo a fin de superar la visión maniquea de Parshley como víctima o verdugo.

\section{EL ORIGINAL Y EL ENCARGO DE TRADUCCIÓN: PARSHLEY Y SUS CIRCUNSTANCIAS}

El segundo sexo, ensayo existencialista arriesgado en el que se analiza en profundidad la condición femenina en las sociedades occidentales, fue un gran éxito de ventas en una Francia todavía tradicional, potenciado también por su postura rompedora. Hablamos, por tanto, de un "succès de scandale" (Bogic, 2009: 4), que se publicó en dos entregas: Les faits et les mythes y L'expérience vécu. Por aquella fecha, la esposa de Alfred Knopf, presidente de una modesta editorial estadounidense, se encontraba en París en busca de nuevos títulos que publicar al otro lado del Atlántico. A pesar de su reticencia inicial, las cifras de ventas en Francia y las opiniones positivas por parte de un grupo de lectores reducido al que entregó el manuscrito a modo de prueba, inclinaron la balanza hacia la compra de los derechos del libro para su traducción y ulterior difusión.

Uno de los más entusiastas receptores iniciales fue el que se convertiría, posteriormente, en el traductor de todo el trabajo, el zoólogo Howard W. Parshley. A diferencia de la visión equivocada de Alfred, que concebía la obra como un tratado sobre el sexo en la línea de American 
Sexual Mores de Kinsey, Parshley manifestó, tras leer la primera parte de la obra de Beauvoir que el libro era: "a profound and unique analysis of woman's nature and position, eminently reasonable and witty, and it surely should be translated, together with the second volume, if the quality is maintained." (a través de Gillman, 1988). A pesar de su especial sensibilidad hacia el tratado, la elección del traductor parece haber sido inspirada por su formación científica y especialización en reproducción, especialmente evidente, tras la publicación en 1933 de su obra The Science of Human Reproduction: Biological Aspects of Sex. Sin embargo, no se cuestionaron como elementos clave en la elección del candidato su nula experiencia como traductor o su formación académica como entomólogo, que nada tenía que ver con la filosofía.

En su valiosa tesis doctoral, Anna Bogic (2009: 17) relata, a través de las misivas intercambiadas entre traductor y editor, que, para más inri, la primera tarea que se le encomendó a Parshley fue seleccionar fragmentos que deberían eliminarse en la versión anglosajona por motivos de venta. Asimismo, el tijeretazo no llevaba manual de instrucciones cualitativo y se dejaba a discreción del zoólogo. Este arduo condicionante provocó, entre otras cuestiones, agrias críticas sobre la calidad del texto inglés a partir de los 80, como comprobaremos en el siguiente epígrafe, y evidencia, por otro lado, el peso que el "iniciador" (Nord, 1991: 8-10) o "mecenas" (Lefevere, 1997: 25-40) tienen en el proyecto de traducción.

En más de una ocasión, ese esfuerzo de síntesis fue objeto de discusión entre los diferentes actantes de la versión inglesa, puesto que la perspectiva del traductor, como reescritor sumido en la ingente empresa de trasvasar texto de más de mil páginas en justos plazos de entrega, daba cuenta del respeto hacia la naturaleza del original frente a la perspectiva mercantilista y ramplona de los revisores de la editorial. Como muestra, un botón:

I have found only two or three short passages that I would want to cut, but there are many that I think should be condensed more or less drastically. Altogether I estimate that hardly sixty pages could be saved in this way. This is not much of a reduction, but with the best will in the world I am unable to apply your injunction: 'cut, slash,' without eliminating what seems to me to be valuable and interesting (carta de Parshley al editor recogida en Gillman, 1988).

Fueron muchos los interrogantes que se planteó el traductor en el proceso de trasvase, más allá de las omisiones de las que, aunque posteriormente renegara, Beauvoir estuvo al tanto. Sus dudas sobre terminología novedosa ( $p$.e. "l'alterité") no obtuvieron respuesta por parte de la autora, quien no parecía muy pendiente del proyecto por su actitud 
"poco cooperativa" según Parshley (Bogic, 2009: 54). La presión de la entrega, que dejó al traductor sin períodos de descanso, repercutió en su estado de salud: sufrió dos infartos, el segundo de los cuales fue mortal. A ello hay que añadir que, como hombre de la América de los 50 , con su propio "habitus" y, a pesar de que compartía la liberación sexual a la que aludía la filósofa en su obra, muchas de las novedosas ideas de Beauvoir, especialmente las relacionadas con el matrimonio, le removían ideológicamente:

\begin{abstract}
"I have not been too happy, myself, about the author's treatment of family life, and I was often tempted to soften her descriptions or insert qualifying footnotes. And the same with many of her remarks about 'conjugal' love and so on. But I had no difficulty in thinking of many actual examples of everything she described, and I thought she was presenting a point of view that needs airing." (carta a Abraham Stone, coautor de A Marriage Manual, recogida en Gillman, 1988).
\end{abstract}

Por tanto, nos parece obvio que el análisis crítico de la traducción de Parshley no solo debe tratar el producto final sino que debe enmarcarlo, como hacemos en la primera parte del presente estudio, en la intrahistoria del proceso de elaboración en donde el traductor no solo se ve sometido a los dictados del TO, a sus limitaciones personales de tipo lingüístico, cognitivo o ideológico, sino también, a las cortapisas de la industria editorial, los ritmos extenuantes de trabajo y la dificultad de acceso a la escritora para recrear un nuevo lenguaje feminista en inglés.

\title{
2. LAS CRÍTICAS: HACIA UNA NUEVA TRADUCCIÓN
}

A pesar de que, en EE. UU. el número de ventas fue elevado justo en el momento del lanzamiento de El segundo sexo, la obra de Beauvoir no representó un revulsivo feminista a la altura de La mística de la feminidad de Betty Friedan, publicada una década después. Junto a otros factores, como el nuevo contexto social de los 60 , la nueva situación laboral de la mujer o el tratamiento de la idiosincrasia americana de esta segunda escritora, algunas voces críticas culparon a la deficiente traducción al inglés de este hecho.

La primera autora que abrió la veda de la demonización de la obra de Parshley fue Margaret Simons, en su artículo de 1983 "The silencing of Simone de Beauvoir: guess what is missing from the Second Sex". En este trabajo, calculaba que un $10 \%$ del libro había sido amputado en el texto inglés, especialmente en la parte de historia de mujeres, y que el existencialismo sartreano se había aguado a través de interpretaciones incorrectas. Se tachaba al traductor de sexista y se le declaraba culpable de 
herir de muerte a un tratado que carecía de sentido y continuidad por haber eliminado los intertextos y referencias filosóficas.

A esta perspectiva se unieron Elizabeth Fallaize (1999, cuya obra fue editada en 2002) y, por supuesto, una Toril Moi (2002) especialmente combativa. La primera realizó un estudio más detallado del capítulo sobre el matrimonio en El segundo sexo, cotejándolo con el original y delineando una metodología que, al centrarse en un corpus más delimitado, es la que vamos a emplear, en lo que compete a los errores, en nuestro análisis sobre la prostitución. Aparte de los efectos sobre los cortes y eliminación de citas textuales, detectó cambios en el tono del mensaje en la traslación. Por su parte, Moi rastreó el libro en busca de los pasajes que le habían valido una fama de inconsistente a Beauvoir como filósofa y, por ende, al movimiento feminista en general. Según la autora, en un contexto de mayor interés por su obra, era de justicia retraducir el ensayo puesto que, en cada una de las páginas de la versión inglesa podía identificar errores (Moi 2002: 1006). La investigadora cargaba las tintas contra los editores y su rechazo a llevar a cabo esta empresa y combinaba el examen textual con el relato del proceso editorial y traductor que comentamos en el apartado anterior. Además de su estudio sobre la terminología existencialista no identificada por Parshley y, por ende, mal traducida, se detenía especialmente en el capítulo sobre la maternidad. Al igual que Fallaize, Moi empleaba una parte del compendio como estudio de caso, que corresponde con nuestra perspectiva en el siguiente epígrafe, en el que no haremos tanto hincapié en la jerga filosófica sino más en la visión lingüística e ideológica de los errores y en sus efectos así como en la concepción de Parshley como mediador.

Finalmente, hemos de indicar que los esfuerzos de estas académicas y de otras más, ${ }^{1}$ dieron sus frutos y, en 2009, se publicó la segunda y esperada traducción íntegra y revisada de la obra de Beauvoir realizada por dos profesoras de inglés del Institut d'Études Politiques de París, Constance Borde y Sheila Malovany-Chevallier. Su periplo de tres años de trabajo es relatado en la nota del traductor que, a modo de prólogo, precede al texto, para dar cuenta del proceso de elaboración, de documentación y el método de traslación empleado, a saber, el filológico, en el que la preservación del estilo del original se convierte en la piedra angular:

When the text is an opus like The Second Sex, whose impact on society was so decisive, the task of bringing into English the closest version possible of Simone de Beauvoir's voice, expression, and mind is greater still (Borde y Malovany-Chevallier en Beauvoir, 2011: 16).

\footnotetext{
1 Bogic (2009) destaca la labor de investigación de Deirdre Bair (1987), Yolanda Astarita Patterson (1992), Sheryl Englund (1992 y 1994) y Anna Alexander (1997).
} 
Sin embargo, a pesar de que fue la primera versión fidedigna del ensayo francés, las traductoras no han escapado a las críticas por su mayor tendencia a emular la estructura del original (Moi 2010: 3-6) y por una supuesta menor fluidez frente al cuarteado texto del 1953. Comprobamos que, nuevamente, la labor traductora se pone en tela de juicio y que se desarrolla bajo la presión de una opinión pública caldeada y escrutadora tras décadas de espera.

\section{PROStitutas Y hetAIRAS}

Al igual que Fallaize (la femme mariée) y Moi (la mère), hemos elegido como objeto de estudio un capítulo de la segunda parte del segundo volumen de la obra de Beauvoir, a saber, Prostituées et Hétaïres (1949b: 246-275). Hemos descartado el primer ejemplar, Les faits et les mythes, que fue mutilado especialmente y sobre cuya traducción han corrido ríos de tinta, y nos hemos inclinado por el siguiente, La expérience vécue, en el que, tras el éxito de la primera edición, Parshley consiguió que el editor abriera el puño de la condensación impuesta. ${ }^{2}$ Por otro lado, elegimos un texto más breve para analizarlo en profundidad; en él, se aúnan los dos estigmas del sujeto descrito: la naturaleza femenina y la prostitución.

Con el cotejo a tres, TO-TM1-TM2, pretendemos señalar los aciertos y errores de la primera traducción, el efecto de los últimos y sus implicaciones ideológicas, así como comprobar de primera mano la calidad del tratamiento terminológico del oficio más antiguo del mundo en la versión contemporánea de Borde y Malovany-Chevallier (utilizamos la reedición de Vintage Books de 2011).

\subsection{Errores del TM1}

\section{Omisiones}

Como se ha mencionado anteriormente, una realidad apriorística que se desprende de los condicionantes del encargo de traducción por parte del mecenas es la exigencia de síntesis y eliminación de material para facilitar la venta de la obra de Beauvoir. Las omisiones quedan al arbitrio de Parshley y no se marcan en el texto meta, por lo que la versión manipulada en inglés se hace pasar por fidedigna. La mayor parte de las mismas se corresponde con citas textuales, referencias o autores eludidos. Veamos algunos ejemplos:

\footnotetext{
2 "My entire attitude toward the book has changed and I am now quite persuaded that this is one of the handful of greatest books on sex ever written. For this reason, you may be sure, I am unlikely to demand further cuts." (Carta de Harold Strauss a Parshley de 1951 tras la publicación de la primera parte de la obra en inglés. Texto extraído de Gillman: 1988).
} 


\begin{tabular}{|c|c|c|}
\hline \multicolumn{3}{|c|}{ Autores } \\
\hline TO & TM1 & TM2 \\
\hline $\begin{array}{l}\text { "L'hetaîrisme, dit } \\
\text { Morgan, suit lhumanité } \\
\text { jusque dans sa civilisation } \\
\text { comme une obscure } \\
\text { ombre portée sur la } \\
\text { famille » }\end{array}$ & $\begin{array}{l}\text { Marriage, as we have } \\
\text { seen, is directly correlated } \\
\text { with prostitution, which, it } \\
\text { has been said, follows } \\
\text { humanity from ancient to } \\
\text { modern times like a dark } \\
\text { shadow over the family }\end{array}$ & 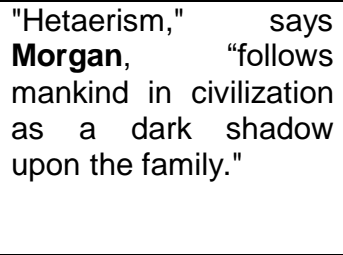 \\
\hline $\begin{array}{l}\text { Et Mandeville dans un } \\
\text { ouvrage qui fit du bruit: } \\
\text { « Il est évident qu'il existe } \\
\text { une nécessité de sacrifier } \\
\text { une partie de femmes } \\
\text { pour conserver l'autre et } \\
\text { pour prévenir une saleté } \\
\text { d'une nature plus } \\
\text { repoussante. " }\end{array}$ & $\begin{array}{l}\text { And it has often been } \\
\text { remarked that the } \\
\text { necessity exists of } \\
\text { sacrificing one part of the } \\
\text { female sex in order to save } \\
\text { the other and prevent } \\
\text { worse troubles. }\end{array}$ & $\begin{array}{l}\text { And Mandeville, in a } \\
\text { very popular book, } \\
\text { said: "It is obvious that } \\
\text { some women must be } \\
\text { sacrificed to save } \\
\text { others and to prevent } \\
\text { an even more abject } \\
\text { filth." }\end{array}$ \\
\hline
\end{tabular}

En los casos referidos, la supresión de la autoría no puede justificarse por motivos de espacio ni exotismo en las referencias, como se le ha atribuido a Beauvoir, tildada de feminista exclusivamente "francesa" o "europea": tanto Morgan ${ }^{3}$ como Mandeville ${ }^{4}$ son autores anglosajones. Por otro lado, la versión de la cita de este último se dulcifica en la traducción: la recreación al francés "une saleté d'une nature plus repoussante" aparece en el texto de Parshley carente de expresividad: "worse troubles". La prosa de El segundo sexo está plagada de referencias y frases de terceros puesto que, precisamente, la argumentación de Beauvoir se nutre y adquiere rigor a través de los hallazgos y nociones de otros autores. Aunque se identifican errores y alusiones vagas en el original, ${ }^{5}$ consideramos innecesarias estas omisiones al tiempo que perniciosas para el estudio de los andamiajes de su tratado.

Asimismo, cabe mencionar que los testimonios obviados en inglés se compensan con el estilo indirecto, lo que reduce el énfasis del original o, en el peor de los casos, deja atrás datos relevantes. Veamos un extracto de la descripción de las situaciones en las que las prostitutas pierden la virginidad

\footnotetext{
${ }^{3}$ La obra de la que extrae la cita es Research in the Lines of Human Rights from Savagery through Barbarium to Civilization (1877) del antropólogo estadounidense Lewis Henry Morgan. ${ }^{4}$ Mandeville escribió A Modest Defence of Public Stews en 1724.

${ }^{5}$ La traductora de Le Deuxième Sexe al español, Alicia Martorell, encabeza la bibliografía que recopila de forma previa al texto del primer volumen con un fragmento de la correspondencia de Beauvoir a Parshley en la que se disculpa por no poder recuperar las referencias a las obras inglesas que consultó y su imposibilidad de encontrar los pasajes citados en el ensayo (Bair: 1990, a través de Martorell en Beauvoir, 1999a: 35).
} 
y otro en el que manifiestan su iniciación homosexual. En aras de la brevedad recogemos dos de las nueve experiencias narradas por el doctor Bizard (Beauvoir, 1949b: 250) en el primer caso y una de las tres del L. Faivre (Beauvoir, 1949b: 254), en el segundo.

\begin{tabular}{|c|c|c|}
\hline \multicolumn{3}{|c|}{ Citas textuales } \\
\hline TO & TM1 & TM2 \\
\hline $\begin{array}{l}\text { (...) R... déflorée à } 17 \text { ans } \\
1 / 2 \text { par un jeune homme } \\
\text { qu'elle n'avait jamais vu et } \\
\text { qu'elle avait par hasard } \\
\text { rencontré chez un } \\
\text { médecin du voisinage } \\
\text { qu'elle était allée chercher } \\
\text { pour sa soeur malade, } \\
\text { qui l'a ramenée en auto } \\
\text { pour qu'elle soit plus vite } \\
\text { rentrée et qui en réalité, } \\
\text { après avoir eu ce qu'il } \\
\text { voulait d'elle, l'a plantée } \\
\text { là en pleine rue.(..) } \\
\text { S... déflorée à } 14 \text { ans par } \\
\text { un jeune homme qui } \\
\text { l'attira chez lui sous le } \\
\text { prétexte de lui faire } \\
\text { connaître sa soeur. Le } \\
\text { jeune homme en réalité } \\
\text { n'avait pas de soeur mais } \\
\text { il avait la syphilis et } \\
\text { contamina la fillette. (...) }\end{array}$ & $\begin{array}{l}\text { The details of individual } \\
\text { cases given in such } \\
\text { reports show how } \\
\text { frequently and under what } \\
\text { varied conditions girls and } \\
\text { young women yield to } \\
\text { casual strangers, new } \\
\text { acquaintances, and older } \\
\text { relatives, in apparent } \\
\text { ignorance of possible } \\
\text { consequences or } \\
\text { indifferent to them. }\end{array}$ & $\begin{array}{l}\text { R...., deflowered at } \\
\text { seventeen and a half by a } \\
\text { young man she had never } \\
\text { seen whom she had met } \\
\text { by chance at the doctor's, } \\
\text { where she had gone to } \\
\text { get the doctor for her } \\
\text { sick sister; he brought } \\
\text { her back by car so that } \\
\text { she could get home more } \\
\text { quickly, but in fact he left } \\
\text { her in the middle of the } \\
\text { street after getting what } \\
\text { he wanted from her. (...) } \\
\text { S...., deflowered at } \\
\text { fourteen by a young man } \\
\text { who drew her to his house } \\
\text { under the pretext that he } \\
\text { wanted her to meet his } \\
\text { sister. The young man in } \\
\text { reality did not have a } \\
\text { sister, but he had syphilis } \\
\text { and contaminated the girl. } \\
\text { (...) }\end{array}$ \\
\hline $\begin{array}{l}\text { L. Faivre a fait, en 1931, } \\
\text { une enquête sur } 510 \\
\text { jeunes prostituées; il a } \\
\text { trouvé que (...) II cite } \\
\text { (avec leur orthographe) } \\
\text { les extraits de lettres } \\
\text { suivants: } \\
\text { Suzanne, 17 ans. Je me } \\
\text { suis livré à la } \\
\text { prostitution avec } \\
\text { surtout des prostituées. } \\
\text { Une qui m'a garder } \\
\text { longtemps, était très } \\
\text { jalouse, aussi j'ai quitté } \\
\text { la rue de... (...) }\end{array}$ & $\begin{array}{l}\text { In a study [nota al pie con } \\
\text { la referencia] of } 510 \text { young } \\
\text { prostitutes, it was found } \\
\text { (..) A number of these } \\
\text { girls said they had been } \\
\text { debauched by other } \\
\text { women, and some of them } \\
\text { prostituted themselves to } \\
\text { females. } \\
\text { [Omision de los } \\
\text { testimonios] }\end{array}$ & $\begin{array}{l}\text { L. Faivre made a study in } \\
1931 \text { of } 510 \text { young } \\
\text { prostitutes; he found that } \\
\text { (...) He cites (with their } \\
\text { spelling) extracts of the } \\
\text { following letters: } \\
\text { Suzanne, seventeen. I } \\
\text { gave myself to } \\
\text { prostitution, especially } \\
\text { with women prostitutes. } \\
\text { One of them who kept } \\
\text { me for a long time was } \\
\text { very jealous, and so I } \\
\text { left that street. (...) }\end{array}$ \\
\hline
\end{tabular}


Podemos comprobar que la casuística de la primera fila, que simplifica Parshley en la traducción, adolece de exhaustividad porque obvia un aspecto importante en algunos de los relatos: el engaño del hombre en situaciones de debilidad (como la convalecencia de una hermana), la crueldad del trato (abandono en mitad de la calle) y la trágica transmisión de enfermedades venéreas. En la segunda cuestión, elimina las escrituras de las prostitutas que, aparte de objetos, representan sujetos con voz propia en el capítulo de nuestro estudio. Más allá de las implicaciones estilísticas de esta forma de narrar por el amortiguamiento del efecto vivaz y auténtico de las declaraciones, los argumentos de Beauvoir pierden fuelle al suprimir el trabajo cualitativo de los autores referenciados. En la traducción de Borde y Malovany-Chavellier los errores ortográficos no se reflejan, por lo que, frente al original, se sacrifica la espontaneidad.

Para finalizar este epígrafe, nos gustaría subrayar una elisión que apunta más a la censura que al condicionante espacial $o$ al hecho de prescindir de referencias galas o europeas: se silencia un icono del cine estadounidense como ejemplo de "hetaira" contemporánea.

\begin{tabular}{|c|c|c|}
\hline \multicolumn{3}{|c|}{ Censura de culturema TM1 } \\
\hline TO & TM1 & TM2 \\
\hline $\begin{array}{l}\text { Même la star, privée } \\
\text { d'appui masculin, voit pâlir } \\
\text { son prestige: quittée par } \\
\text { Orson Welles, c'est avec } \\
\text { un air souffreteux } \\
\text { d'orpheline que Rita } \\
\text { Hayworth a erré à travers } \\
\text { l'Europe avant d'avoir } \\
\text { rencontré Ali Khan. }\end{array}$ & $\begin{array}{l}\text { Even the star, deprived of } \\
\text { masculine support, sees } \\
\text { her prestige grow dim. }\end{array}$ & $\begin{array}{l}\text { (...) deprived of masculine } \\
\text { support, even the movie } \\
\text { star sees her prestige } \\
\text { fade: abandoned by } \\
\text { Orson Welles, Rita } \\
\text { Hayworth wandered over } \\
\text { Europe like a sickly } \\
\text { orphan until she found Aly } \\
\text { Khan. }\end{array}$ \\
\hline
\end{tabular}

\subsection{Cambios de sentido}

A pesar de las críticas vertidas contra Parshley por su dudoso dominio del francés, el capítulo no muestra cambios de sentido sustanciales (a excepción del falso amigo échec>check que veremos más adelante) si bien su traducción destila, en ciertos fragmentos, una visión patriarcal y moralista de la sociedad en la que vive inmerso y en la que se crió como "varón". Como muestra, un botón:

\begin{tabular}{|c|c|c|}
\hline TO & TM1 & TM2 \\
\hline $\begin{array}{l}\text { (...) il y a un grand nombre } \\
\text { de jeune filles qui se } \\
\text { laissent déflorer par le } \\
\text { premier venu et qui } \\
\text { trouveront ensuite nature }\end{array}$ & $\begin{array}{l}\text { (...) a great many young girls } \\
\text { let themselves be deflowered } \\
\text { by the first comer and } \\
\text { thereafter find it natural to } \\
\text { yield to anyone. }\end{array}$ & $\begin{array}{l}\text { Many girls let } \\
\text { themselves be } \\
\text { deflowered by the first } \\
\text { comer and then find it } \\
\text { natural to give }\end{array}$ \\
\hline
\end{tabular}




\begin{tabular}{|c|c|c|}
\hline $\begin{array}{l}\text { de se donner au premier } \\
\text { venu. }\end{array}$ & & $\begin{array}{l}\text { themselves ro } \\
\text { anyone who comes } \\
\text { along }\end{array}$ \\
\hline $\begin{array}{l}\text { Plus de la moitié disaient } \\
\text { s'être données par amour. }\end{array}$ & $\begin{array}{l}\text { More than half said they gave } \\
\text { themselves for love }(\ldots)\end{array}$ & $\begin{array}{l}\text { More than half said } \\
\text { they gave } \\
\text { themselves out of } \\
\text { love. }\end{array}$ \\
\hline $\begin{array}{l}\text { (...) les autres avaient } \\
\text { consenti par ignorance }\end{array}$ & $\begin{array}{l}\text { They others had yielded } \\
\text { through ignorance }\end{array}$ & $\begin{array}{l}(\ldots) \text { the others } \\
\text { consented } \\
\text { out of ignorance. }\end{array}$ \\
\hline $\begin{array}{l}\text { (...) elles avaient consenti } \\
\text { avec indifférence }(\ldots)\end{array}$ & They yielded indifferently & $\begin{array}{ll}\ldots) \quad \text { they } & \text { had } \\
\text { consented } & \text { with } \\
\text { indifference }(. . .) & \\
\end{array}$ \\
\hline $\begin{array}{l}\text { La maladie pousse souvent } \\
\text { à la prostitution la femme } \\
\text { (...). De même la } \\
\text { naissance d'un enfant. }\end{array}$ & $\begin{array}{l}\text { Sickness often drives into } \\
\text { prostitution women (..) } \\
\text { Bearing an illegitimate child } \\
\text { has the same result. }\end{array}$ & $\begin{array}{l}\text { Illness often leads to } \\
\text { prostitution as the } \\
\text { woman (...) So it is } \\
\text { with the birth of a } \\
\text { child. }\end{array}$ \\
\hline
\end{tabular}

En los cuatro primeros ejemplos del cuadro anterior observamos cómo, casi de manera sistemática, el acto de 'entregarse por propia voluntad' (se donner) o 'mantener relaciones sexuales consentidas con alguien' (consentir) es traducido por el verbo "to yield" en la primera versión de la obra. Según el diccionario monolingüe Merriam Webster, este verbo implica 'rendirse al control físico de otra persona, claudicar' lo que, si bien mantiene el tono machista de "se donner" acentúa su tono pasivo (la mujer se rinde, cede, no es ella, como sujeto, la que "se da a sí misma"). Por su parte, "consentir" subraya la libertad de la mujer que muestra conformidad con la propuesta sexual, que se infiere como premisa al consentimiento, por lo que la traducción más antigua se aleja en mayor medida del núcleo de significado original.

En el último de los ejemplos del cuadro, Parshley incluye un adjetivo moralista, "illegitimate" como complemento de "child" ausente en el original y en la actual traducción al inglés. Realmente se desconoce en el contexto original si los vástagos cuya manutención arrastra a las mujeres a la prostitución son siempre fruto de relaciones extramatrimoniales.

\begin{tabular}{|l|l|l|}
\hline TO & TM1 & TM2 \\
\hline $\begin{array}{l}\text { (...) ce respect commence } \\
\text { à faire sérieusement } \\
\text { échec à l'oppression. }\end{array}$ & $\begin{array}{l}\text { beginning definitely to to } \\
\text { check the oppresion. }\end{array}$ & $\begin{array}{l}(\ldots) \text { this respect begins } \\
\text { seriously to bring a halt } \\
\text { to oppression. }\end{array}$ \\
\hline $\begin{array}{l}\text { Dans l'ensemble, } \\
\text { cependant, la femme } \\
\text { travaille «à froid ". }\end{array}$ & $\begin{array}{l}\text { In general, however, the } \\
\text { woman is 'cold' when } \\
\text { professionally at work. }\end{array}$ & $\begin{array}{l}\text { In general, however, the } \\
\text { woman works "cold." }\end{array}$ \\
\hline
\end{tabular}




\begin{tabular}{l|l|l|} 
En vérité, ce n'est pas la & It is not, in fact, Beauty & In fact, it is not Beauty \\
Beauté qui engendre le & that arouses desires; but \\
désir; mais la théorie & the justification of ouses desire: but \\
the Platonic theory of \\
platonicienne de \\
l'amour propose à la la \\
lubricité d'hyprocrites \\
lustifications.
\end{tabular}

En la tabla anterior incluimos otros desvíos en la traducción de Parshley: en el primer ejemplo, se produce un cambio total de sentido por la similitud formal entre échec (acabar con) y check (comprobar), es decir, por causa de un falso amigo; en el segundo, la expresión francesa, que tiene una doble lectura, marcada por las comillas, se simplifica en el texto meta más antiguo: del original se desprende que la prostituta no solo mantiene una actitud fría cuando trabaja sino que ejerce su profesión "en frío", sin preparación ni deseo; en el último, la inversión de los elementos sintácticos en la traducción del zoólogo estadounidense lleva al equívoco: indica, incorrectamente, que la teoría de la belleza platónica justifica la lascivia. En realidad, Beauvoir matiza y ve en el precepto una coartada filosófica para la lujuria: "en realidad, no es la Belleza la que engendra el deseo, pero la teoría platónica del amor aporta justificaciones hipócritas para la lubricidad" (traducción de A. Martorell en Beauvoir, 1999: 368).

\subsection{Variación lingüística y connotaciones}

Junto a la conjunción de retazos de diferentes géneros discursivos (novela, notas de un estudio, relatos de las prostitutas, diálogos) que convergen en el ensayo de Beauvoir y que, en ocasiones, se eliminan o adelgazan por "espacio" en la traducción de Parshley, destacamos el empleo de eufemismos para aludir a las meretrices. A veces, como se puede comprobar en los tritextos seleccionados, el tono del mensaje se modifica en el TM1 mediante el uso de apelativos directos (prostitutes y harlots frente a filles) o coloquiales y peyorativos (whores). En otras, la metáfora (au trottoir), como otra forma de expresión oblicua del campo semántico de la prostitución, se traduce por su sentido directo (prostitution):

\begin{tabular}{|l|l|l|}
\hline TO & TM1 & TM2 \\
\hline $\begin{array}{l}\text { On a marqué entre autres } \\
\text { qu'une grande partie de } \\
\text { « filles " se recontraient } \\
\text { parmi les servantes }\end{array}$ & $\begin{array}{l}\text { It noteworthy, for one } \\
\text { thing that a large } \\
\text { proportion of harlots are } \\
\text { former domestic servants. }\end{array}$ & $\begin{array}{l}\text { It has been noted that, } \\
\text { many "girls" were } \\
\text { once servants. }\end{array}$ \\
\hline $\begin{array}{l}\text { Les moralistes bien } \\
\text { pensants répondent en } \\
\text { ricanant que les récits }\end{array}$ & $\begin{array}{l}\text { Right-thinking moralists } \\
\text { reply sneeringly that the } \\
\text { sob-stories of whores }\end{array}$ & $\begin{array}{l}\text { Right-thinking moralists } \\
\text { respond sneeringly that } \\
\text { the pitiful accounts of }\end{array}$ \\
\hline
\end{tabular}




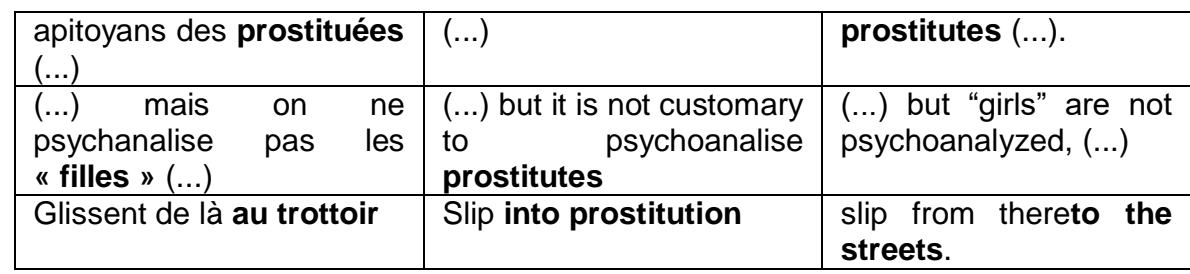

4. Las técnicas de PaRshley fRente a las de Borde y MalovanyCHAVELLIER

Hasta el momento hemos comprobado, por el estado de la cuestión y nuestro estudio de caso, que la primera traducción de Le deuxième sexe hacía aguas por muchos flancos. No obstante, hemos de admitir que el reescritor, aun sin experiencia en el campo, muestra conciencia de su labor, busca la naturalidad en la medida de sus posibilidades y tiene en mente al lector meta. Como prueba de ello, recogemos a continuación nombres de personajes conocidos en el mundo francés que, lejos de pasar por la tijera de Parshley, son completados o sustituidos con paráfrasis que permiten a un público más general que el actual, con mayor formación o, incluso especializado, acceder a referencias de la cultura meta.

\begin{tabular}{|l|l|l|}
\hline TO & TM1 & TM2 \\
\hline $\begin{array}{l}\text { C'est pour trouver des } \\
\text { «protecteurs "que Nana } \\
\text { monte sur la scène. }\end{array}$ & $\begin{array}{l}\text { Zola's Nana appears on the } \\
\text { stage in order to find } \\
\text { 'protectors'. }\end{array}$ & $\begin{array}{l}\text { Nana goes onstage } \\
\text { to find herself a } \\
\text { "protector." }\end{array}$ \\
\hline Nino de Lenclos & $\begin{array}{l}\text { Ninon de Lenclos: } \\
\text { seventeenth-century } \\
\text { woman of wit and beauty. }\end{array}$ & Ninon de Lenclos \\
\hline Ce rôle d'Egérie & $\begin{array}{l}\text { The role of Egeria, the } \\
\text { trusted counsellor of men. }\end{array}$ & the role of Egeria \\
\hline $\begin{array}{l}\text { Les changements } \\
\text { sociaux et économiques } \\
\text { ont aboli le type des } \\
\text { Blanche d'Antigny. }\end{array}$ & $\begin{array}{l}\text { Social and economic } \\
\text { changes have abolished this } \\
\text { flamboyant type. }\end{array}$ & $\begin{array}{l}\text { Social and economic } \\
\text { changes abolished } \\
\text { the Blanche } \\
\text { d'Antigny types. }\end{array}$ \\
\hline
\end{tabular}

A pesar de que nos parece injusto, por la ingente empresa que emprendió y las circunstancias del encargo primigenio, quemar en plaza pública a Parshley, hemos de admitir, sin lugar a dudas, que la traducción de 2009 del capítulo analizado supera los escollos de la primera: no tiene cortes, ofrece una sintaxis fiel a la de la autora (ejemplo 1), allana la expresión retorcida de Parshley (ejemplo 2) y pule la vaguedades con términos precisos (ejemplos 3, 4 y 5): 


\begin{tabular}{|c|c|c|}
\hline \multicolumn{3}{|c|}{ Sintaxis y puntuación fiel al TO (1) } \\
\hline $\mathrm{TO}$ & TM1 & TM2 \\
\hline $\begin{array}{l}\text { (...) aussi longtemps } \\
\text { qu'existeront la police, la } \\
\text { prostitution, il y aura des } \\
\text { policiers, des prostituées. } \\
\text { D'autant qu'en moyenne ces } \\
\text { métiers rapportent plus que } \\
\text { beaucoup d'autres. }\end{array}$ & $\begin{array}{l}\text { (...) as long as a police } \\
\text { force and prostitution } \\
\text { exist, there will be } \\
\text { policemen } \\
\text { prostitutes, and } \\
\text { especially as these } \\
\text { occupations pay better } \\
\text { than many others. }\end{array}$ & $\begin{array}{l}\text { (...) as long as there are } \\
\text { police and prostitution, } \\
\text { there will be policemen } \\
\text { and prostitutes. } \\
\text { Especially because } \\
\text { these professions are, } \\
\text { on average, more } \\
\text { lucrative than many } \\
\text { others. }\end{array}$ \\
\hline
\end{tabular}

En este tritexto observamos cómo la puntuación del original se mantiene en el TM2 mientras que se altera, generando una oración más extensa, en el TM1.

\begin{tabular}{|c|c|c|}
\hline \multicolumn{3}{|c|}{ Paráfrasis ilegítima (2) } \\
\hline TO & TM1 & TM2 \\
\hline $\begin{array}{l}\text { Dans la prostitution, le désir } \\
\text { masculin, étant non singulier } \\
\text { mais spécifique, peut } \\
\text { s'assouvir sur n'importe quel } \\
\text { corps. }\end{array}$ & $\begin{array}{l}\text { In prostitution, male } \\
\text { desire can be satisfied } \\
\text { on no matter what body, } \\
\text { such desire being } \\
\text { specific but not } \\
\text { individualized as to } \\
\text { object }\end{array}$ & $\begin{array}{l}\text { In prostitution, } \\
\text { masculine desire can be } \\
\text { satisfied on any body as } \\
\text { it is specific and not } \\
\text { individual. }\end{array}$ \\
\hline
\end{tabular}

Advertimos en la muestra precedente que la expresión se vuelve compleja y excesivamente descriptiva en el TM1 (paráfrasis ilegítima). Por su parte, en los siguientes fragmentos queda patente la mayor concreción y exhaustividad de la nueva traducción.

\begin{tabular}{|c|c|c|}
\hline \multicolumn{3}{|c|}{ Precisión en TM2 (3, 4 y 5) } \\
\hline TO & TM1 & TM2 \\
\hline $\begin{array}{l}\text { (...) d'un « abattage » qui } \\
\text { souvent s'accompagne d'une } \\
\text { fatigue physique épuisante, } \\
\text { d'un passe rapide, d'un } \\
\text { "couché ", ou des } \\
\text { relations suivies avec un } \\
\text { client familier. }\end{array}$ & $\begin{array}{l}\text { (...) a rapid and fatiguing } \\
\text { passage from one } \\
\text { customer to another, or } \\
\text { of repeated relations } \\
\text { with a familiar client. }\end{array}$ & $\begin{array}{l}\text { "mass turnover," often } \\
\text { physically exhausting, a } \\
\text { quick trick, or regular } \\
\text { relations with a familiar } \\
\text { client. }\end{array}$ \\
\hline Mystifications & Deceptive offers of work & Mystifications \\
\hline $\begin{array}{l}\text { Soumettent à lui avec } \\
\text { d'autant plus de docilité. }\end{array}$ & $\begin{array}{l}\text { Submits to him all the } \\
\text { more readily. }\end{array}$ & $\begin{array}{l}\text { submit to him even more } \\
\text { docilely. }\end{array}$ \\
\hline
\end{tabular}




\section{CONCLUSIONES}

Tras el estudio de caso de "Prostitutas y hetairas" de El segundo sexo de Simone de Beauvoir concluimos lo siguiente:

Hemos comprobado que la primera traducción del capítulo está cuarteada como el resto de los dos volúmenes, a decir de los trabajos publicados anteriormente, aunque en menor medida. La eliminación de citas textuales de prostitutas, nombre de autores o datos cualitativos de estudios referenciados empobrece el ensayo y su línea argumentativa. Asimismo, impide acceder a las situaciones denigrantes de las mujeres protagonistas desde sus circunstancias y vivencias íntegras narradas en estilo directo. No obstante, no todos los intertextos se omiten y no hemos logrado identificar un criterio homogéneo de selección del material "indultado". Como se deduce de las misivas intercambiadas entre el traductor y sus editores, la mutilación textual fue una condición impuesta y, por tanto, salvo en la elección del material suprimido, la responsabilidad no puede recaer totalmente en los hombros fatigados de Parshley.

Se advierte una traducción sexista en la descripción de la pérdida de la virginidad de las prostitutas y en la inclusión innecesaria del adjetivo "illegitimate" como complemento de los hijos de las meretrices; no obstante, consideramos que esas connotaciones han podido pasar inadvertidas por Parshley dado el contexto del capítulo, su propia crianza y la naturaleza del encargo de traducción, urgente, laborioso y sin un feedback productivo con la autora. Estas pinceladas no ensombrecen del todo una reescritura que, a pesar de sus irregularidades, logra transmitir, a nuestro entender, el mensaje principal.

En ciertos fragmentos, Parshley se muestra poco exhaustivo con la terminología, estilo y variación lingüística. Parece más pendiente del fondo que de la forma. No obstante, revela conciencia del trasvase cultural que supone su tarea y se visibiliza en el texto con la inclusión de paráfrasis explicativas de personajes propios de la historia francesa, a fin de facilitar la comprensión del público general meta.

Definitivamente la traducción del 2009 suple las carencias de su predecesora y apuesta por la fidelidad al texto original, léxica y sintácticamente. La obsesión por rehabilitar la autoría perdida de Beauvoir se manifiesta, además, en la puntuación textual. La mirada puesta en el TO aparece descrita en el prólogo de las traductoras, quienes dirigen su trabajo hacia un público más exigente, especializado y escrutador. Consideramos injustas las críticas dirigidas hacia ellas al comprobar, en cada uno de los fragmentos seleccionados, la correcta reformulación y mejora de la traducción del 1953, haciendo alarde de destreza y empleando un vocabulario más preciso y contemporáneo. 
REFERENCIAS BIBLIOGRÁFICAS

BAIR, Deirdre, Simone de Beauvoir, A Biography. New York: Summits Books, 1990.

BEAUVOIR, Simone, El segundo sexo. La experiencia vivida (vol. 2). Madrid: Cátedra, 1999.

—, El segundo sexo. Los hechos y los mitos (vol. 1). Madrid: Cátedra, 1999.

—, Le deuxième sexe (vol. 1). Gallimard, 1949.

—, Le deuxième sexe (vol. 2). Gallimard, 1949.

—, The Second Sex. New York: Lowe \& Brydone, 1956.

_- The Second Sex. New York: Vintage Books (Random House), 2011.

BogIc, Anna, Rehabilitating Howard M. Parshley: A Socio-historical Study of the English Translation of Beauvoir's Le deuxième sexe, with Latour and Bourdieu. Tesis Doctoral. Ottawa: University of Ottawa, 2009.

Dictionnaire de français Larousse. Disponible en línea en: http://www.larousse.fr/ [Fecha de consulta: 26 de enero de 2016].

FALLAIZE, Elizabeth, "Le destin de la femme au foyer: traduire 'la femme mariée' de Simone de Beauvoir". En: Cinquantenaire du Deuxième sexe, Christine Delphy and Sylvie Chaperon (eds.). Paris: Éditions Syllepse, 2002, pp. 468-474.

Gillman, Richard, "The Man Behind the Feminist Bible", En: The New York Times, 22 de mayo de 1988.

LEFEVERE, André, Traducción, reescritura y la manipulación del canon literario. Salamanca: Ediciones Colegio de España, 1997.

Merriam-Webster Dictionary. Disponible en línea en: http://www.merriamwebster.com/ [Fecha de consulta: 26 de enero de 2016].

Mol, Toril, "The Adulteress Wife". En: London Review of Books, 3, 6, 2010, pp. 3-6.

—, "While We Wait: The English Translation of The Second Sex." En: Signs 27, 4, 2002, pp. 1005-1035.

NoRD, Christiane, Text Analysis in Translation. Theory, Methodology, and Didactic Application of a Model for Translation-Oriented Text Analysis. Amsterdam: Rodopi, 1991.

SimONS, Margaret, "The Silencing of Simone de Beauvoir: Guess What's Missing from The Second Sex". En: Women's Studies International Forum 6, 5, 1983, pp. 559-564. 